\title{
Phase Transformations in Binary Alloys under High Pressure
}

\author{
V.F. Degtyareva \\ Institute of Solid State Physics, Russian Academy of Sciences \\ Chernogolovka, Moscow district, 142432, Russia
}

\begin{abstract}
High-pressure studies of binary alloy systems display some specific features connected with composition referring as a variable parameter. In a two-component system under pressure there are observed transformations, which do not occur in a one-component system. In particular, there is a formation of a single phase from a mixture of two phases or a decomposition of a uniform phase into two phases of different compositions. Alloys of In and Sn with neighbouring elements give us examples of these kinds of transformations. Studies on binary alloys with application of pressure provide experimental evidences that the alloy composition or electron concentration is one of the main factor for structural phase stability following from Hume-Rothery rule.
\end{abstract}

PACS numbers: $61.50 . \mathrm{Ks}, 62.50 .+\mathrm{p}, 61.66 . \mathrm{Dk}$

\section{Introduction}

High-pressure studies are mainly focused on elements or on compounds already existing at ambient pressure, e.g. on one-component or quasi-one-component systems. Investigations on binary and multi-component systems under pressure are largely confined to given alloy composition. For a two-component system along with two thermodynamic parameters, pressure and temperature, one has to consider an additional variable parameter - composition. This offers for a binary system an additional degree of freedom in phase equilibria and allows the occurrence of phase transformations under pressure, which do not occur in a one-component system.

A binary compound of a stoichiometric composition in high-pressure studies is usually approached to a quasi-one-component system, however this consideration could be applied with certain restrictions. It is often observed that when according to thermodynamic equilibrium conditions a binary compound should decompose, 
the transformation does not occur because of kinetic hindrance of diffusion process. The observed state of the sample is therefore metastable. With an increase in pressure the melting temperature usually increases together with the diffusion barrier that hinders the attainment of the equilibrium state. This behaviour should be taken into account when one considers phase transformations of stoichiometric compounds under pressure.

At first glance one should expect that application of pressure to binary alloy systems would produce a more complicated picture in phase equilibria comparing to that at the ambient pressure because of:

- polymorphic transitions of constituents;

- phase transitions of ambient-pressure intermediate phases and compounds;

- decomposition of ambient-pressure compounds;

- formation of new intermediate high-pressure phases and their transformation on further pressure increase.

Systematic studies on binary alloys under pressure were initiated by Ponyatovskii with the "pressure quenching" method (Ref. [1]) and continued in situ under pressure (Refs. [2-8]). These studies show that the common picture of $T-x$ phase equilibria for binary alloy systems can be simplified by introducing an additional variable - pressure. Three-dimensional $T-P-x$ phase diagrams for homologous binary systems turned out to be similar to each other, but shifted along the pressure axis. Missing at ambient pressure intermediate phases can appear in a binary alloy system under high pressure.

Under pressure the group IV and V elements of semiconducting/semimetallic type transform to a metallic state. In the binary alloy systems of these elements with the neighbouring metals the phase equilibria evolve under pressure from semiconductor/semimetal-metal type to metal-metal type. The main specific features of these changes are as following:

- extension of solid solutions based on constituents or ambient-pressure intermediate phases of a metallic type;

- decomposition of stoichiometric compounds or intermediate narrow-range phases;

- formation of new intermediate high-pressure phases with metallic type of bonding;

- occurrence of phase sequences along valence electron concentration displaying a decrease in packing density and coordination number;

- determination of the alloy phase stability predominantly by a factor of the valence electron concentration in spirit of the known Hume-Rothery or electron phases.

Studies on binary alloys of $s p$ metals with the relatively simple electron configuration are of special interest. A binary alloy of the elements neighbouring in the periodic table with minimal differences in atomic sizes and electronegativity can be considered as a "modelling element" with the valency as a variable param- 
eter. This system is very suitable for theoretical analysis on the structure stability. The high-pressure phases obtained in In and Sn based alloys give experimental evidences for Brillouin-zone-Fermi-sphere (BZ-FS) fitting as a crucial factor of the phase stability for these nearly-free-electron metals. With this respect high pressure is used as a tool to analyse phase stability in modelling metals with a "variable valency". These studies are important in relation to electron transfers under pressure observed in several groups of elements such as alkali and alkali-earth elements, lanthanides and actinides.

In this paper some features of phase stability in binary alloys of $s p$ metals will be outlined on the base of recent high-pressure studies performed in diamond anvil cells with the use of synchrotron radiation (Refs. [2-8]).

\section{Formation of new intermediate phases in binary alloys under pressure}

Phase equilibria in a system of $C$ components existing in $P$ phases are determined by the Gibbs phase rule: at given pressure and temperature as variables, the number of degrees of freedom, $F$, is $F=C-P+2$. A two-component system therefore has one degree of freedom more than a one-component system. When the pressure is varied at a fixed temperature, in the system with $C=2$ three phases can coexist in equilibrium. This allows transformations from a two-phase mixture into a single-phase state and inversely - a decomposition of a stoichiometric compound or an intermediate phase into two phases of different compositions.

\subsection{The Sn-based binary alloys with In and $\mathrm{Hg}$}

A simple hexagonal (sh) phase exists at ambient conditions only in Sn-based alloys with $\mathrm{In}$ and $\mathrm{Hg}$ in quite narrow composition regions corresponding to the electron concentration of $\approx 3.8$ el./atom [9, 10]. Phase diagrams of In-Sn and $\mathrm{In}-\mathrm{Hg}$ at normal pressure are given in Fig. 1 together with plots of the phase sequences observed under pressure [4-7].

The interest to high-pressure studies of these alloys first arises from the occurrence of sh phase in pure group IV elements Si and Ge under pressure, whilst it is not found in pure $\mathrm{Sn}$. The light group IV elements $\mathrm{Si}$ and $\mathrm{Ge}$ show some common features in their high-pressure transformations with the general trend: diamond $(c F 8) \rightarrow$ white tin $(t I 4) \rightarrow$ simple hexagonal $(h P 1) \rightarrow$ close packed structures $(h P 2, c F 4)[11-15]$.

Besides these high-symmetry structures, also some intermediate low-symmetry structures have been observed. The white tin phase transforms to sh through an orthorhombic Imma structure, oI4 [16, 17]. The transformation from sh to hcp occurs in Si through a low-symmetry intermediate phase, Si-VI [12], determined as oC16, space group Cmca [18], recently found also in Ge [19]. 


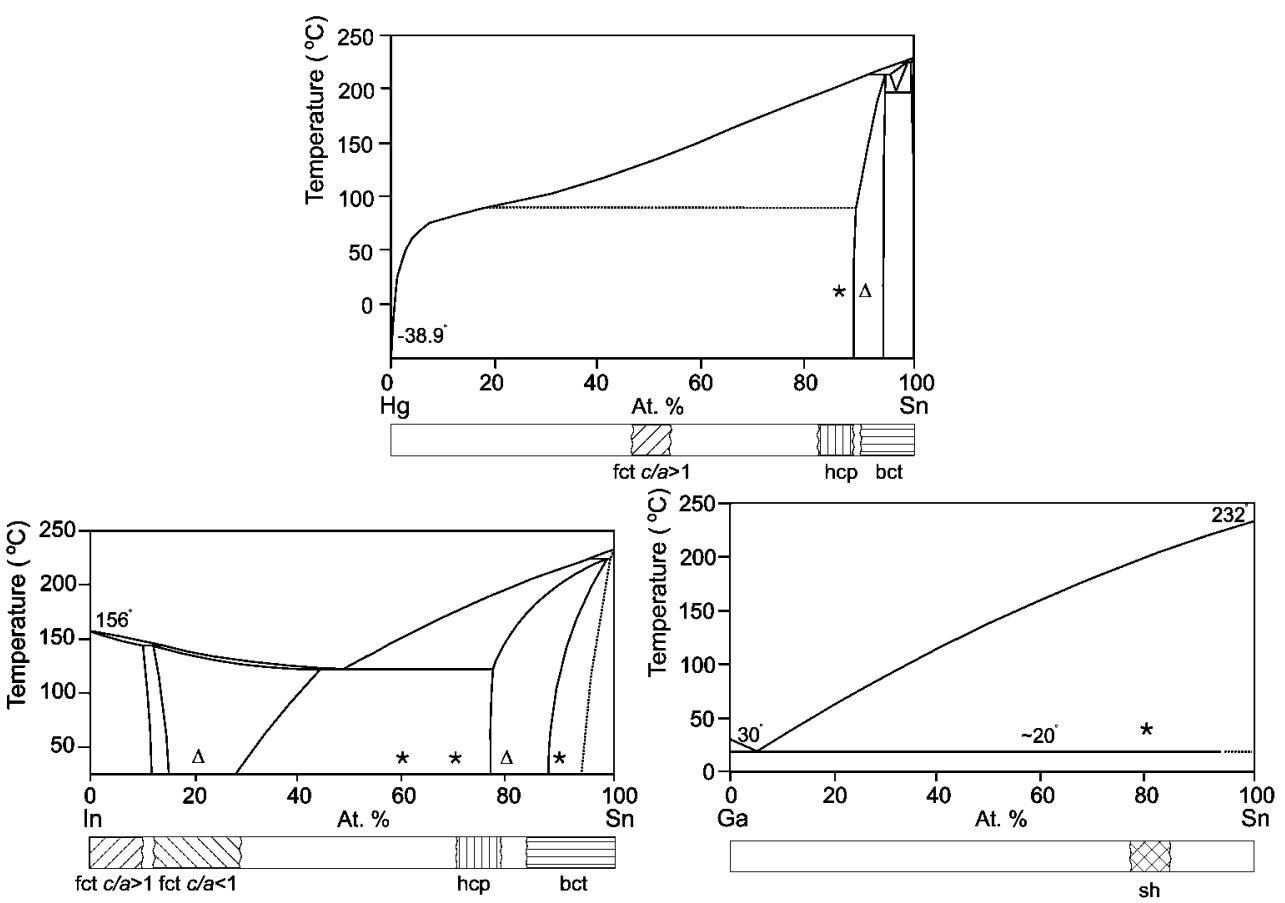

Fig. 1. Phase sequences along the composition in the $\mathrm{Hg}-\mathrm{Sn}, \mathrm{In}-\mathrm{Sn}$ and $\mathrm{Ga}-\mathrm{Sn}$ alloy systems. Ambient pressure phase boundaries are based on the data of $[9,10]$. The stars and triangles on the phase diagrams indicate the compositions of the alloys studied in our work. The plots below the diagrams show schematically regions of phase stability under pressure and the phase sequences along the composition.

The heavier group IV element Sn displays a different structural sequence under pressure [20] with diamond $(c F 8) \rightarrow$ white tin $(t I 4) \rightarrow$ body-centered tetragonal $(t I 2) \rightarrow$ body-centered cubic $(c I 2)$, omitting $h P 1$ and the related distorted structures.

Our recent studies on the sh phase in Sn-In alloys show that starting with a single-phase alloy at ambient pressure we obtain a two-phase mixture on the pressure increase $[5,7]$. Thus the sh phase in the $\operatorname{In}_{20} \mathrm{Sn}_{80}$ alloy transforms to hcp and bct phases above $10 \mathrm{GPa}$ and relative amounts of these phases in the alloy changes with pressure, so that only the hcp phase exists above $25 \mathrm{GPa}$. The studies on other compositions of In-Sn alloys neighbouring the sh phase (Sn with 10 and 30 at.\% In) allow for observation of single-phase fields with bet and hcp, as shown in Fig. 2.

$\mathrm{Hg}-\mathrm{Sn}$ alloys with the sh phase show similar behaviour to In-Sn alloys, though in this system the hep phase occurred at much higher pressures around $55 \mathrm{GPa}$ (Ref. $[4,7]$ ). The hcp phase in $\mathrm{In}-\mathrm{Sn}$ and $\mathrm{Hg}-\mathrm{Sn}$ alloys is a new intermediate 


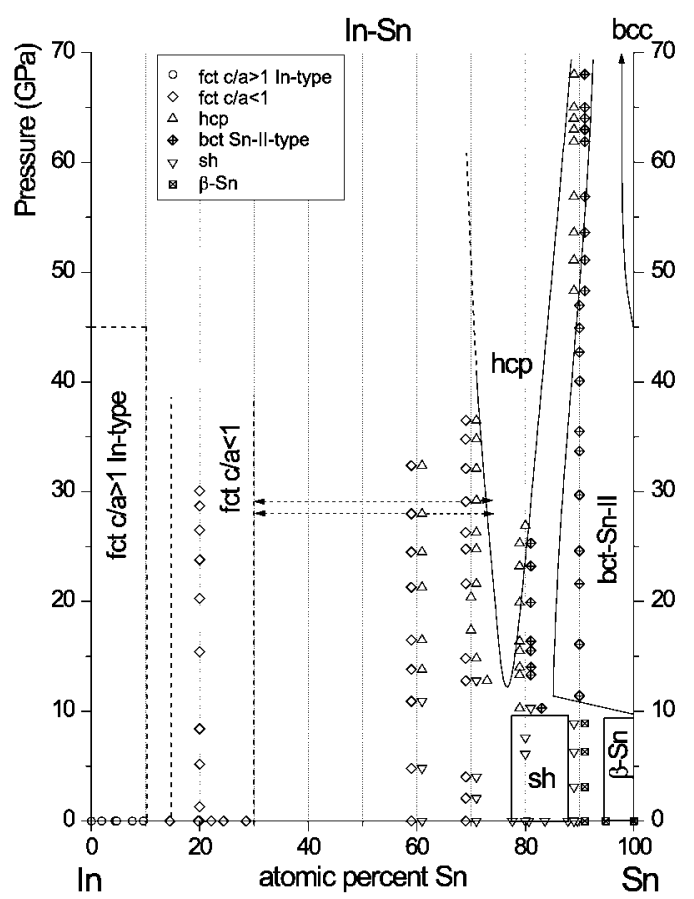

Fig. 2. A $P-x$ diagram of phase regions for the In-Sn alloy system. Different symbols on the phase diagram denote experimental points for the alloys studied, corresponding to different phases observed. Two (or three) symbols at one point indicate a phase-mixture, where the corresponding composition of the phases is shown by horizontal arrows. Phase compositions are determined from atomic volume consideration. For experimental details see Ref. [7].

high-pressure phase in Sn-alloys found experimentally. The sh-to-hcp transformation was recently reported for the alloy $\operatorname{In}_{25} \mathrm{Sn}_{75}$ by the group of Meenakshi and co-workers [22]. These studies point out to the region of stability for the hcp phase near 3.75 el./atom. The sh-to-hcp transformation was also observed in $\mathrm{Al}_{30} \mathrm{Ge}_{70}$ alloy under pressure, when both sh and hcp phases are intermediate high-pressure phases [2].

The bct phase in Sn alloys is related to the high-pressure bct phase in tin (Sn II) [20] and should be regarded as a solid solution based on Sn II. The stability region of the bct solid solution extends to $\approx 15$ at. $\%$ In and to $\approx 10$ at. $\% \mathrm{Hg}$, and these regions tend to narrow with the pressure increase. An interesting feature of these bct phases is a correlation between a degree of tetragonal distortion $(\mathrm{c} / \mathrm{a}$ ratio) and the alloy composition. The axial ratio decreases with a decrease in alloy electron concentration. The possible explanation of this correlation should be accounted for by Brillouin-zone-Fermi-sphere fittings discussed in papers [3, 4]. 


\subsection{The sh phase in the $G a-S n$ alloys}

The Ga-Sn system has a phase diagram of simple eutectic type at zero pressure [9, 10] (Fig. 1). However, in the homologous In-Sn system an intermediate sh phase occurs on the Sn-rich side (around 20 at.\% In) already at zero pressure $[9,10]$. Formation of a similar phase was expected in $\mathrm{Ga}-\mathrm{Sn}$ alloy system under pressure. From the previous studies on binary alloys a "homology rule" was found for $T-P-x$ diagrams, which says that the effect of pressure is often similar to a replacement of one of the constituents by a heavier element from the same group in the periodic table [1].

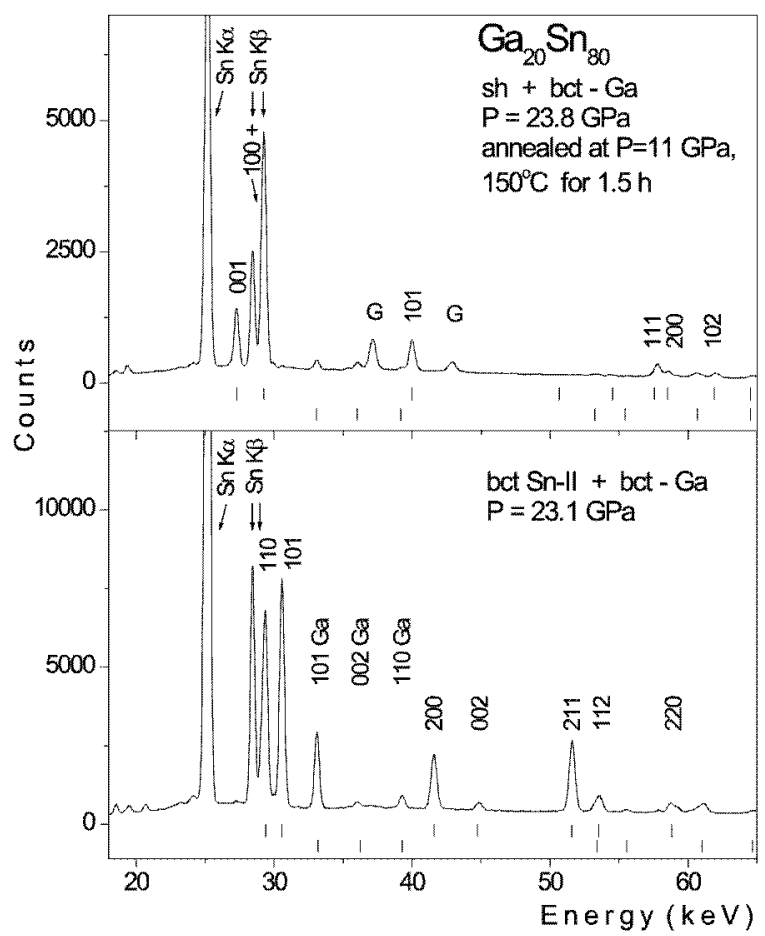

Fig. 3. Energy dispersive diffraction spectra for the $\mathrm{Ga}_{20} \mathrm{Sn}_{80}$ alloy collected with $2 \theta=9.63^{\circ}(E d=7383 \mathrm{keV} \mathrm{pm})$. The pattern at $23.1 \mathrm{GPa}$ corresponds to a two-phase mixture: bct Sn II with $a=355.1(2) \mathrm{pm}, c=330.0(3) \mathrm{pm}, c / a=0.929$ (peaks $h k l$ ) and bct-Ga with $a=265.7(4) \mathrm{pm}, c=407.6(6) \mathrm{pm}$ (peaks $h k l \mathrm{Ga}$ ). After pressure decrease to $11.4 \mathrm{GPa}$ and heating at $150^{\circ} \mathrm{C}$ for $1.5 \mathrm{~h}$ the pattern at $23.8 \mathrm{GPa}$ corresponds to sh with $a=291.5(2) \mathrm{pm}, c=270.7(2) \mathrm{pm}, c / a=0.929$ (peaks $h k l$ ) and minor addition of bct-Ga with $a=266.4(12) \mathrm{pm}, c=410.2(6) \mathrm{pm}$. The calculated peak positions are shown as tick marks below each pattern. " $G$ " denotes diffraction peaks from the gasket material. For experimental details see Ref. [7]. 
We have performed high-pressure studies on $\mathrm{Ga}_{20} \mathrm{Sn}_{80}$ alloy, initially containing $\beta$-Sn and $\mathrm{Ga}$. The pressure increase without heating results in transformations in both components: at pressures around $23 \mathrm{GPa}$ two phases were observed, bct-Ga (In type) and bct-Sn, in agreement with the data for pure components [23, 20] as shown in Fig. 3. We have examined this alloy after heating at $150^{\circ} \mathrm{C}$ for about $1.5 \mathrm{~h}$ under pressure $11.4 \mathrm{GPa}$. A diffraction pattern taken at room temperature at $11.4 \mathrm{GPa}$ after this heating revealed a new phase with an sh structure together with a small amount of bct-Ga diffraction peaks. The lattice parameters for the sh phase are $a=299.5(2) \mathrm{pm}, c=277.9(3) \mathrm{pm}$, and $c / a=0.928$ at $11.4 \mathrm{GPa}$. The composition of the sh phase is close to $15-18$ at.\% Ga. The sh phase is stable in the alloy with pressure increasing up to $\approx 30 \mathrm{GPa}$ reached in this study and decomposes on decreasing pressure.

This result confirms the homology rule for binary alloy systems under pressure. The electron concentration of the sh phase in $\mathrm{Ga}-\mathrm{Sn}$ corresponds to a particular stability region around $3.8 \mathrm{el}$./atom of the sh phases in In-Sn and $\mathrm{Hg}-\mathrm{Sn}$. The structural data for sh in $\mathrm{Ga}-\mathrm{Sn}$ gives the value of the axial ratio which perfectly agrees with the $c / a=0.93$, known for the most sh phases.

\subsection{The In-based binary alloys with $C d$, Sn and $P b$}

The group III element In crystallizes in a body-centered tetragonal (bct) structure, $t I 2$, space group $I 4 / \mathrm{mmm}$, which is a small distortion of the face-centered cubic, fcc, structure. Therefore, it is more convenient to describe it as a face-centered tetragonal, fct, structure with an axial ratio $c / a=1.075$ [24]. Indium remains in this structure under pressure at least up to $50 \mathrm{GPa}$ [23], with slight variation in $c / a$, the fcc phase has not been observed for In.

However a change from fct to fcc occurs by alloying of In with group II metals $\mathrm{Cd}$ or $\mathrm{Hg}$ [24]. The addition of these elements of lower valency decreases $c / a$ of the fct structure resulting in the fcc structure at $\approx 6$ at.\% of $\mathrm{Cd}$ or $\mathrm{Hg}$. $\mathrm{An}$ addition of metals with higher valency, as $\mathrm{Sn}$ or $\mathrm{Pb}$, results in an increase in $c / a$ up to 1.10 . However at concentrations higher than $\approx 12$ at.\% Sn or $\mathrm{Pb} c / a$ jumps from $c / a>1$ to $c / a<1$ [24]. The existence of phase regions fct $c / a>1$ and fct $c / a<1$ is shown in the phase diagram of In-Sn in Fig. 1. The variation of the axial ratio on the alloy composition for tetragonal phases in In alloys is given in Fig. 4.

Our recent high-pressure studies [6] on $\mathrm{Cd}_{10} \operatorname{In}_{90}$ alloy show a fcc-to-fct transition at pressure $\approx 1.5 \mathrm{GPa}$. The axial ratio jumps discontinuously from 1 to 1.04 and then it increases with pressure up to 1.074 at $30 \mathrm{GPa}$ displaying behaviour similar to pure In (see Fig. 4).

Our further studies [6] were performed on the fcc phase in In-Pb alloys existing under ambient conditions at the $\mathrm{Pb}$ content higher than 30 at.\% [24]. The alloys with 40 and 60 at.\% $\mathrm{Pb}$ both show a transition from fcc to fct, whereas $c / a$ jumps from 1 to $c / a<1$ as shown in Fig. 4. With the pressure increase the 


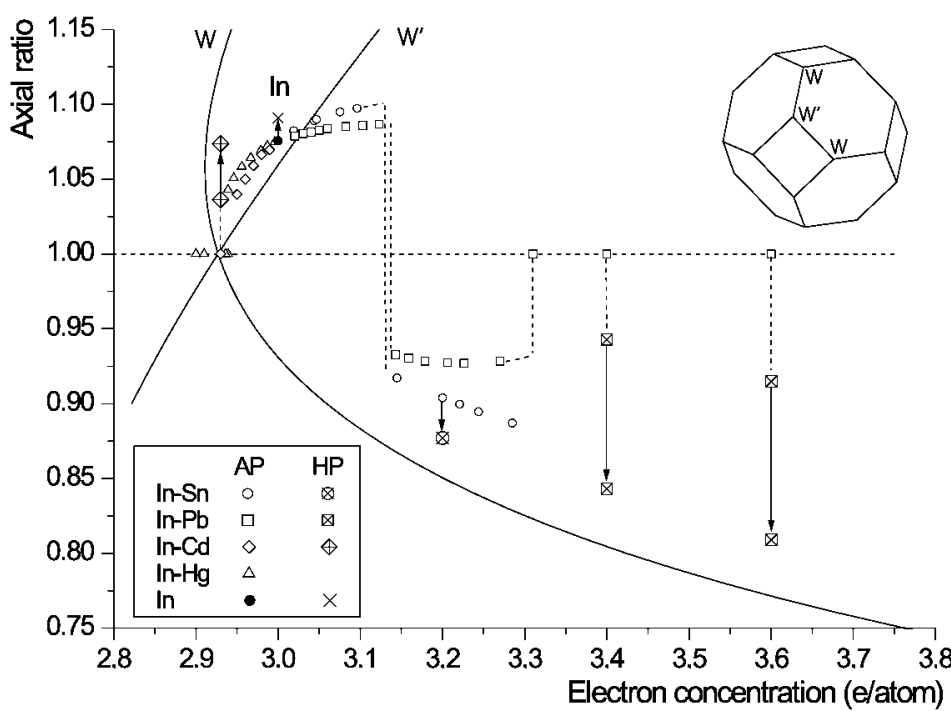

Fig. 4. Axial ratio vs. electron concentrations for fct phases in In-alloys at ambient pressure (open symbols), based on results of Ref. [24], and at high pressure (crossed symbols) from our studies. The full circle and the cross correspond to the data on In at normal and at pressure $23 \mathrm{GPa}$ taken from Ref. [21]. The arrows show the change of $c / a$ under pressure from the value just at the fcc-fct transition to the value at the highest pressure reached $(\approx 30 \mathrm{GPa})$. The full curves represent the calculated distortion $(c / a$ vs. $n$ ) when the Brillouin zone corners of type $W$ and $W^{\prime}$ touch the free electron Fermi sphere determined by $n$. The Brillouin zone of the fcc structure is shown in the upper right corner. For experimental details see Ref. [6].

tetragonal distortion increases, at the same pressure $c / a$ is lower for the higher $\mathrm{Pb}$ content. This result was quite puzzling until the common plot was drawn for tetragonal phases in In-based alloys, Fig. 4.

The studies on $\operatorname{In}_{80} \mathrm{Sn}_{20}$ alloy [6] with the initial phase fct $c / a<1$ also show an increase in tetragonal distortion with the increase in pressure. The observed changes in $c / a$ for this alloy were less pressure dependent, however these correlate to the common trend in the variation of $c / a$ along the electron concentration, Fig. 4. The dependence of the axial ratio on the mean number of valence electrons, $n$, indicates an electronic origin of the tetragonal distortion as will be considered below.

\subsection{The In-Bi alloy system}

In the In-Bi alloy system $[9,10]$ there are at ambient pressure three intermediate compounds $\mathrm{In}_{2} \mathrm{Bi}, \mathrm{In}_{5} \mathrm{Bi}_{3}$, and $\mathrm{InBi}$, Fig. 5. All three compounds exist at certain stoichiometric compositions and have crystal structures of site-ordered type. 


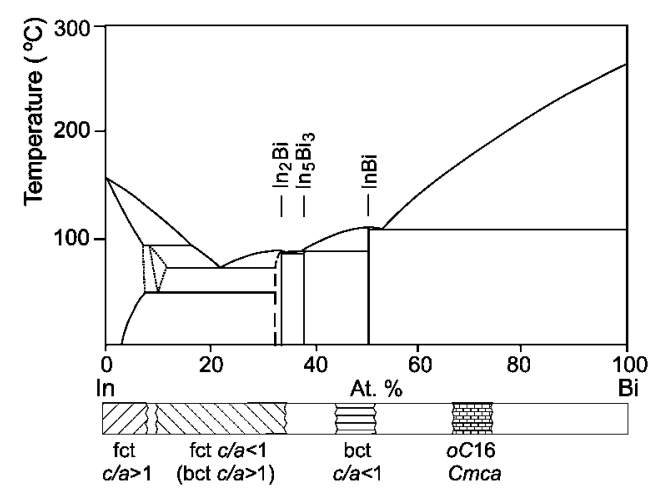

Fig. 5. Comparison of the phase sequences along the composition in the In-Bi alloy systems at the ambient pressure (upper plot), based on data from [9], and at high pressure (lower plot), based on the results from Refs. [8, 25].

The InBi compound was studied by energy-dispersive diffraction with synchrotron radiation in a diamond-anvil cell under pressure up to $75 \mathrm{GPa}$ [3]. Initial tetragonal structure is $t P 4$, space group $P 4 / n m m$, with $c / a=0.95$. First transformation occurs with a strong compression along $c$ to a similar structure with $c / a=0.65$ and then to a tetragonal body-centered structure, bct, which is of a random (disordered) type. The bct phase shows an increase in $c / a$ from 0.91 to 0.96 in the pressure range from 20 to $\approx 70 \mathrm{GPa}$. Some evidences was obtained for a transition to a cubic body-centered structure, bcc. The behaviour of the III-V compound InBi is similar to that of the isoelectronic group IV element Sn. The important peculiarity of this similarity is the same upper value of the axial ratio of the bct structure attained in both cases.

The $\mathrm{In}_{5} \mathrm{Bi}_{3}$ compound crystallises in a tetragonal structure with lattice parameters $a=854.4 \mathrm{pm}$ and $c=1268 \mathrm{pm}$, with 32 atoms in the unit cell, space group $14 / m c m$ [24]. Recent studies under pressure [8] shown that this structure remained stable at least up to $25 \mathrm{GPa}$. However, after annealing of the $\operatorname{In}_{5} \mathrm{Bi}_{3} \mathrm{sam}$ ple under pressure $15 \mathrm{GPa}$ at $150^{\circ} \mathrm{C}$ for 4 hours the diffraction pattern changes pointing out a phase transition (see Fig. 6).

No solution for this pattern was found by assuming this state to be a single-phase state and therefore an attempt to account for by a two-phase mixture was made. A tentative solution was found as a two-phase mixture where both phases are body-centered tetragonal, space group $14 / \mathrm{mmm}$, differing by the axial ratio. We assign one phase with the axial ratio $c / a=0.92$ as bct $c / a<1$, and the other phase with $c / a=1.13$ as bct $c / a>1$. The atomic volumes of both these phases have slightly different values which allow an estimation (on the ground of Vegard's law) the difference in the phase compositions as about 10 at.\%. This observation implies that the $\mathrm{In}_{5} \mathrm{Bi}_{3}$ compound decomposes into two phases of different composition: one is above and one is below of the initial $\operatorname{In}_{5} \mathrm{Bi}_{3}$ com- 


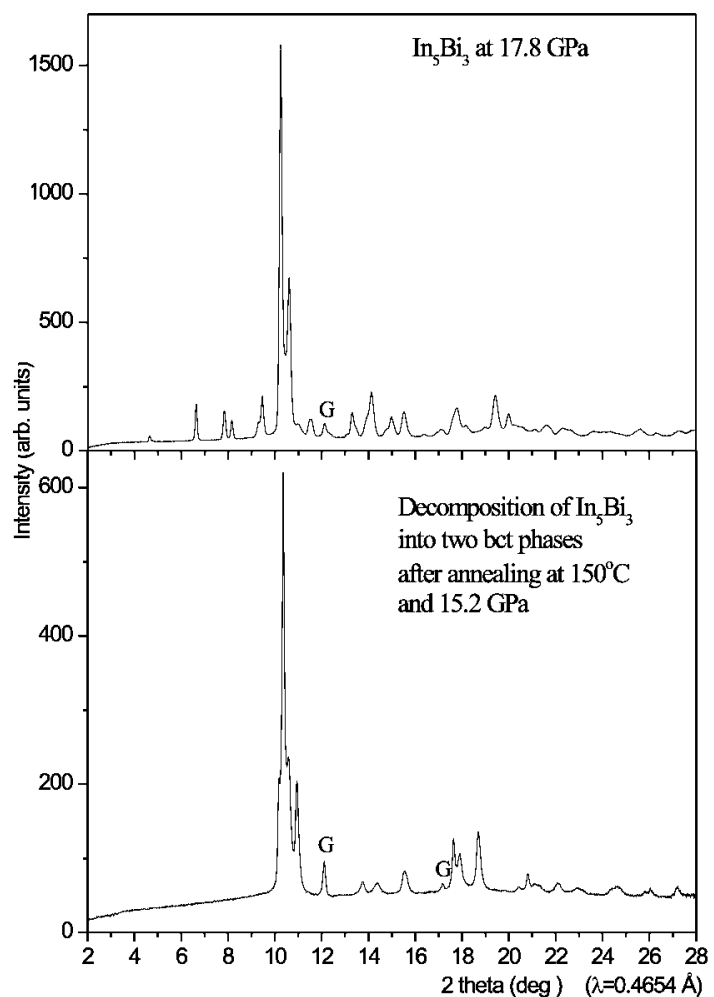

Fig. 6. Angle dispersive diffraction spectra (integrated profiles) from the $\operatorname{In}_{5} \mathrm{Bi}_{3}$ compound (upper) at $17.8 \mathrm{GPa}$ without heating; (lower) at $15 \mathrm{GPa}$ after annealing at $150^{\circ} \mathrm{C}$, $4 \mathrm{~h}$, corresponding to a decomposition on a two-phase mixture with bct $c / a<1$ and bct $c / a>1$. "G" denotes peaks from the gasket material. For experimental details see Ref. [8].

position. This transformation is reversible, and the $\operatorname{In}_{5} \mathrm{Bi}_{3}$ compound is recovered on decompression.

In the Bi-rich region of the In-Bi alloy system several intermediate phases were obtained by "pressure quenching" [1], as a simple hexagonal (sh) phase and an orthorhombically distorted sh phase. A complex phase was found near the composition $\operatorname{In}_{30} \mathrm{Bi}_{70}$ with the crystal structure determined recently as $\mathrm{Cmca-oC16}$ [25], related to high-pressure forms of $\mathrm{Si}$ and $\mathrm{Ge}[18,19]$. The region of $\mathrm{Cmca-oC16}$ phase should be extended at certain $P, T$-conditions up to pure Bi, because Bi IV phase is assumed to be similar to $C m c a-o C 16$ structure $[26,25]$.

Under high pressure a sequence of metallic randomly occupied phases can be outlined with quite extended regions of stability as shown schematically in the lower plot of Fig. 5. The structure of the phases in this sequence is determined by the factor of the number of valence electrons as for so-called Hume-Rothery 
phases. The In-rich region of the In-Bi alloy system displays the following structural sequence under high pressure:

$$
\text { fct } c / a>1 \rightarrow \text { fct } c / a<1(=\text { bct } c / a>1) \rightarrow \text { bct } c / a<1 \text {. }
$$

This sequence is consistent with that for $\mathbf{I n - S n}$ alloy system under ambient and high pressure shown in Fig. 1.

There is much similarity in the high-pressure phases of binary and high-pressure forms of group IV elements. This implies these phases to be common representatives of polyvalent $s p$ metals and alloys, rather than particular characteristics of certain elements or groups.

\section{The Bain path fcc-bcc and Brillouin zone concept}

Tetragonal distortions from fcc to bcc structures represent a so-called Bain path. Both structures can be described with the common cell, whether body-centered tetragonal, bct, with $c / a$ equal to $\sqrt{2}$ and 1 , or face-centered tetragonal, fct, with $c / a$ equal to 1 and $1 / \sqrt{2}$ for $\mathrm{fcc}$ and bcc, respectively. To understand the stability of the tetragonal phase against the cubic one, one should consider two contributions to the crystal energy: (1) the electrostatic Ewald term and (2) the electronic band-energy contribution due to Fermi-sphere-Brillouin-zone interaction. The electrostatic energy calculated for a common tetragonal structure along the deformation from fcc to bcc has two minima corresponding to $c / a=1$ (fcc) and $c / a=1 / \sqrt{2}$ (bcc) [27]. This indicates that Ewald term favours the high symmetry cubic structures and the tetragonal distortion is caused by other terms.

Attempting to understand the stability of the tetragonal phases in In-alloys, Svechkarev [28] has supposed a lowering in the crystal energy, when BZ corners touch the FS. The plot of $c / a$ vs. electron concentration, $n$, calculated for $\mathrm{BZ}$ touching FS by corners of type $W$ and $W^{\prime}$, is presented in Fig. 4 (solid curves). A contact of all $W$-type corners with FS results in fcc phase, as it appears at $n=2.94$, i.e. by alloying of In with $\approx 6$ at.\% $\mathrm{Cd}$ or $\mathrm{Hg}$.

For the In alloys with the electron concentration $n<3.12$, the axial ratio follows the upper branch of $c / a$ vs. $n$, as shown in Fig. 4. For the In alloys with the electron concentration $n>3.15$, the tetragonal distortion follows the lower branch with $c / a<1$. This phase exists at ambient pressure in the range from $\approx 15$ to $\approx 30$ at. $\% \mathrm{Sn}$ or $\mathrm{Pb}$. Our observation of the fcc-to-fct transition on In alloys with 40 and 60 at.\% $\mathrm{Pb}$ indicates that pressure causes an extension of the fct region towards $\mathrm{Pb}$. The values of $c / a$ in this extended region follow the Svechkarev plot $c / a$ vs. $n$, providing a new evidence for this model of BZ-FS fitting.

According to this model, one can consider the stability of either fcc or fct as determined by a balance between electrostatic and electronic band energy terms. The latter contribution to the crystal energy appears to become more significant under pressure, stabilising the tetragonal distortion. Following a linear extrapolation of the experimental data toward bcc, the latter is expected to be stable at 
$n \approx 4$ el./atom, as the plot $c / a$ vs. $n$ crosses $c / a=1 / \sqrt{2}$ at $n=4.2$. The bcc structure is realised under pressure in the heavy group IV elements $\mathrm{Sn}$ and $\mathrm{Pb}$ $[20,29]$. However, a tetragonal distortion occurs in Sn under pressure before the transition to bcc, with the axial ratio reaching $c / a=0.96$ (0.68 in the fct setting) [20]. Similar tetragonal distortion of bcc has been observed under pressure in the isoelectronic compound $\mathrm{InBi}$, with the same axial ratio [3]. The dashed line in Fig. 7 extending from this point at $n=4$ towards lower electron concentration represents tetragonal distortions for bct in the Sn-rich alloys with $\mathrm{In}$ and $\mathrm{Hg}$ under pressure $[4,5,7]$.

Thus the common plot of the Bain path from fec to bcc as a function of $c / a$ vs. $n$ represents a linear behaviour of $c / a$ in the middle part with strong deviations from linearity by approaching both fcc and bcc.

Recent observation of decomposition under pressure of the $\operatorname{In}_{5} \mathrm{Bi}_{3}$ compound $(n=3.75)$ and formation of two bct phases with different $c / a$ [8] corresponds to the proposed model. Axial ratios of both bet phases fall onto the plot just in the region of the $c / a$ jump from $c / a>1$ to $c / a<1$ for bct phases, Fig. 7 .

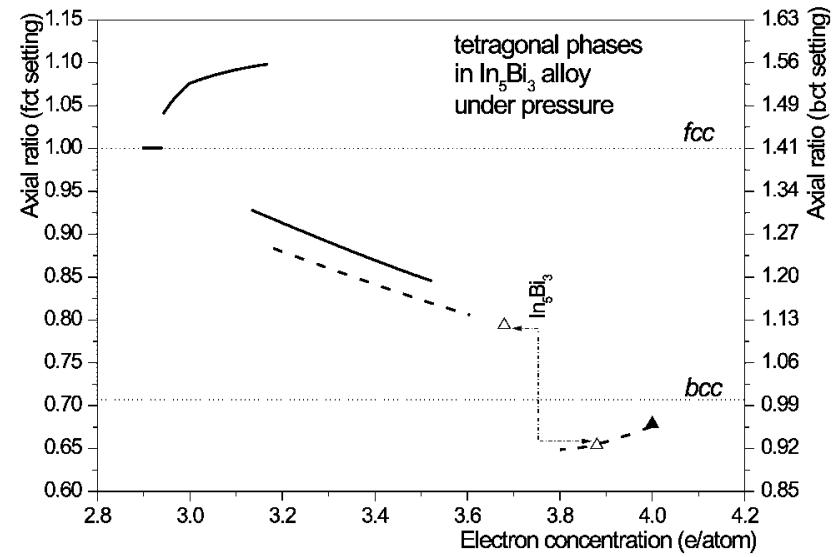

Fig. 7. A representative plot for axial ratios of the tetragonal phases for In-Sn and related alloys as a function of electron concentration. Dotted horizontal lines correspond to $c / a$ values for $f c c$ and bcc structures. The solid lines represent average values of axial ratios for In alloys with $\mathrm{Cd}, \mathrm{Hg}, \mathrm{Sn}, \mathrm{Pb}$, and $\mathrm{Bi}$ at ambient pressure, whether stable [24] or metastable [30]. The dashed lines represent axial ratios observed at high pressure for the alloys $\mathrm{In}-\mathrm{Sn}, \mathrm{In}-\mathrm{Pb}$ [6] (middle branch), and $\mathrm{Sn}-\mathrm{Hg}$, Sn-In, In-Bi (lower branch) $[3-5,7,8]$. The dot-dashed vertical line indicates the composition of the initial $\operatorname{In}_{5} \mathrm{Bi}_{3}$ compound, and horizontal arrows indicate its decomposition into two tetragonal phases with different $c / a$ ratios and different compositions, denoted by open triangles. The solid triangle denotes the high pressure bct phase in InBi [3]. 


\section{Conclusion}

High pressure studies on binary alloys show specific transformations like a formation of new intermediate phase from a two-phase mixture and inversely - a decomposition of a homogeneous phase into two phases of different composition. These transformations are allowed for a two-component system, due to an additional variable - composition, which provides for the phase equilibria one degree of freedom more than for a one-component system in accordance with the Gibbs phase rule. For instance, the sh phase in the $\mathrm{In}_{20} \mathrm{Sn}_{80}$ alloy decomposes to hcp and bct phases above $10 \mathrm{GPa}$ and, inversely, the sh phase is formed under pressure from the mixture of $\mathrm{Sn}$ and $\mathrm{Ga}$ in the $\mathrm{Ga}_{20} \mathrm{Sn}_{80}$ alloy. The $\mathrm{In}_{5} \mathrm{Bi}_{3}$ compound was found to decompose under pressure into two bct phases with different $c / a$ and different compositions.

A binary alloy system having at ambient pressure a phase diagram with stoichiometric compounds or narrow-range phases evolves under pressure to a phase diagram with a sequence of metallic phases which are mostly of random or substitutional type with quite extended regions of stability. The composition ranges of high-pressure phases do not relate directly to those of low-pressure compounds or phases.

The structure and stability regions of the high-pressure phases in the binary $s p$ metal alloys are determined mainly by a factor of the electron concentration as for the electronic Hume-Rothery phases.

\section{Acknowledgments}

The author would like to thank Prof. E.G. Ponyatovskii, Prof. W.B. Holzapfel, Dr. F. Porsch and O. Degtyareva for discussions and collaboration. Partial support from the Russian Foundation for Basic Research by the grant No. 01-02-97030 is gratefully acknowledged.

\section{References}

[1] E.G. Ponyatovskii, V.F. Degtyareva, High Press. Res. 1, 163 (1989).

[2] V.F. Degtyareva, F. Porsch, E.G. Ponyatovskii, W.B. Holzapfel, Phys. Rev. B 53, 8337 (1996).

[3] V.F. Degtyareva, M. Winzenick, W.B. Holzapfel, Phys. Rev. B 57, 4975 (1998).

[4] V.F. Degtyareva, O. Degtyareva, M. Winzenick, W.B. Holzapfel, Phys. Rev. B 59, 6058 (1999).

[5] V.F. Degtyareva, O. Degtyareva, W.B. Holzapfel, K. Takemura, Phys. Rev. B 61, $5823(2000)$.

[6] O. Degtyareva, V.F. Degtyareva, F. Porsch, W.B. Holzapfel, J. Phys., Condens. Matter 13, 7295 (2001). 
[7] O. Degtyareva, V.F. Degtyareva, F. Porsch, W.B. Holzapfel, J. Phys., Condens. Matter 14, 389 (2001).

[8] O. Degtyareva, V.F. Degtyareva, J. Phys., Condens. Matter 14, 407 (2001).

[9] T.B. Massalsky, Binary Alloy Phase Diagrams, American Society for Metals, Metals Park, Ohio, 1996.

[10] M. Hansen, K. Anderko, Constitution of Binary Alloys, McGraw Hill, New York 1958, reprinted by Genium Publishing Corp., New York 1989.

[11] J.C. Jamieson, Science 139, 762 (1963).

[12] H. Olijnyk, S.K. Sikka, W.B. Holzapfel, Phys. Lett. A 103, 137 (1984).

[13] J.Z. Hu, I.L. Spain, Solid State Commun. 51, 263 (1984).

[14] Y.K. Vohra, E. Brister, S. Desgreniers, A.L. Ruoff, K.L. Chang, M.L. Cohen, Phys. Rev. Lett. 56, 1944 (1986).

[15] S.J. Duclos, Y.K. Vohra, A.L. Ruoff, Phys. Rev. Lett. 58, 775 (1986).

[16] M.I. McMahon, R.J. Nelmes, Phys. Rev. B 47, 8337 (1993).

[17] R.J. Nelmes, H. Liu, S.A. Belmonte, J.S. Loveday, M.I. McMahon, D.R. Allan, D. Häusermann, M. Hanfland, Phys. Rev. B 53, R2907 (1996).

[18] M. Hanfland, U. Schwarz, K. Syassen, K. Takemura, Phys. Rev. Lett. 82, 1197 (1999).

[19] K. Takemura, U. Schwarz, K. Syassen, M. Hanfland, N.E. Christensen, D.L. Novikov, I. Loa, Phys. Rev. B 62, 10603 (2000).

[20] H. Olijnyk, W.B. Holzapfel, J. Phys. (Paris) Colloq. 45, c8-153 (1984).

[21] K. Takemura, Phys. Rev. B 44, 545 (1991); K. Takemura, H. Fujihira, Phys. Rev. $B$ 47, 8465 (1993).

[22] S. Meenakshi, V. Vijayakumar, B.K. Godwal, S.K. Sikka, Phys. Rev. B 64, 212104 (2001).

[23] O. Schulte, W.B. Holzapfel, Phys. Rev. B 55, 8122 (1997).

[24] W.B. Pearson, A Handbook of Lattice Spacings and Structures of Metals and Alloys, Pergamon Press, Vol. 1, 1964, Vol. 2, 1967.

[25] V.F. Degtyareva, Phys. Rev. B 62, 9 (2000).

[26] J.H. Chen, H. Iwasaki, T. Kikegawa, J. Phys. Chem. Solids 58, 247 (1997).

[27] V. Heine, D. Weaire, in: Solid State Physics, Vol. 24, Academic Press, New York 1970 , p. 459.

[28] I.V. Svechkarev, Zh. Eksp. Teor. Fiz. 47, 961 (1964) [Sov. Phys. JETP 20, 643 (1965)].

[29] C.A. Vanderborgh, Y.K. Vohra, H. Xia, A.L. Ruoff, Phys. Rev. B 41, 7338 (1990).

[30] B.C. Giessen, M. Morris, N.J. Grant, Trans, Metall. Soc. AIME 239, 883 (1967). 